\title{
Padrões de interações mutualísticas entre espécies arbóreas e aves frugívoras em uma comunidade de Restinga no Parque Estadual de Itapuã, RS, Brasil
}

\author{
Adriano Scherer ${ }^{1,2}$, Fabiana Maraschin-Silva ${ }^{1}$ e Luís Rios de Moura Baptista ${ }^{1}$
}

Recebido em 16/03/2006. Aceito em 29/08/2006

\begin{abstract}
RESUMO - (Padrões de interações mutualísticas entre espécies arbóreas e aves frugívoras em uma comunidade de Restinga no Parque Estadual de Itapuã, RS, Brasil). Padrões de interação entre aves frugívoras e espécies arbóreas, com suas variações sazonais, foram determinados em uma mata de Restinga. Foram feitas observações visuais e capturas da avifauna, ao longo de um ano, estimando-se a conectância do sistema mutualístico e o índice de importância das espécies. Também foi elaborada a rede de interações do sistema e feita a análise da variação das interações entre as estações do ano. Foram registradas 18 espécies animais e 11 vegetais interagindo, com uma conectância de 25,3\%. Turdus amaurochalinus e T. rufiventris apresentaram maior número de visitas, interagiram com a maioria das espécies arbóreas e tiveram o maior índice de importância, sendo caracterizadas como as principais dispersoras em potencial. Ocotea pulchella e Myrsine spp. foram registradas com os maiores números de eventos de consumo de frutos pelas aves. No entanto, Ficus organesis interagiu com mais espécies dispersoras, além de ter a maior importância na dieta das aves. Observaram-se variações na quantidade de eventos de frugivoria ao longo do ano, com um aumento significativo na primavera. Além disso, verificou-se um aumento nas espécies frugívoras durante a primavera e verão, bem como no número de espécies arbóreas visitadas nessas estações.
\end{abstract}

Palavras-chave: interação ave-planta, frugivoria, estrato arbóreo, Mata Atlântica

\begin{abstract}
Patterns of mutualistic interactions between tree species and frugivorous birds in a restinga community at Itapuã State Park, Rio Grande do Sul, Brazil). Interaction patterns between frugivorous birds and tree species, with seasonal variations, were determined in a restinga forest. Visual observations and bird captures were performed throughout a year, estimating the connectance of the mutualistic system and the importance index of species. The interaction network of the system was also worked out and seasonal variation in these interactions was analyzed. Eighteen animal species and 11 tree species were recorded as interacting, with a connectance of $25.3 \%$. Turdus amaurochalinus and T. rufiventris had the highest number of visits, interacted with most tree species and had the highest importance index, being characterized as the main potential dispersers. Ocotea pulchella and Myrsine spp. were recorded with the highest numbers of fruit consumption events by the birds. Ficus organensis, however, interacted with more disperser species, besides being most important for the birds' diet. Variations in the number of frugivory events throughout the year were observed, with a significant increase in spring. Moreover, an increase in frugivorous species was verified during spring and summer, as well as in the number of tree species visited during these seasons.
\end{abstract}

Key words: bird-plant interaction, frugivory, tree stratum, Atlantic Forest

\section{Introdução}

A remoção de frutos por animais representa a fase inicial na dispersão de sementes, que possibilita uma maior chance de recrutamento desses propágulos longe de seu local de origem, sendo esses frutos uma fonte de nutrientes para os frugívoros. Esse tipo de mutualismo entre animais e plantas se constitui em uma relação mais complexa do que uma simples troca de vantagens e é influenciado por diversos fatores (Howe \& Smallwood 1982).
Após a remoção dos frutos, o sucesso da dispersão das sementes (longe da planta-mãe) depende diretamente do comportamento alimentar do frugívoro, do processamento do fruto e dos movimentos pós-alimentares (Jordano \& Schupp 2000). O destino das sementes depende se estas são ou não danificadas durante a mandibulação ou digestão (Levey 1987; Corlett \& Lucas 1990) e se elas são descartadas embaixo da copa da planta-mãe ou em sítios adequados (Howe \& Vande Kerckhove 1981; Wheelwright 1991).

\footnotetext{
1 Universidade Federal do Rio Grande do Sul, Programa de Pós-Graduação em Botânica, Av. Bento Gonçalves 9500, Bloco 4, Prédio 43.433, Sala 214, 91501-970 Porto Alegre, RS, Brasil

2 Autor para correspondência: adriano@sisrest.org.br
} 
Em florestas tropicais, pelo menos $50 \%$ e freqüentemente mais de $75 \%$ das espécies arbóreas produzem frutos carnosos adaptados para o consumo por aves e mamíferos (Howe \& Smallwood 1982). Entre os organismos mais importantes na dispersão de sementes, as aves compõem uma parcela significativa da biomassa de vertebrados (Terborgh 1986), além de apresentarem vantagens sobre outros grupos dispersores, no que diz respeito à sua mobilidade e à grande diversidade de espécies.

Mesmo sendo difícil verificar todas as relações existentes entre as espécies de aves e de árvores e o nível de dependência entre elas, algumas espécies se destacam na dieta dos frugívoros, sendo importantes para a manutenção das populações de frugívoros em períodos que outras espécies não estão frutificando (Howe 1977; 1981; Silva et al. 2002). Estas interações complexas são importantes na dinâmica do ecossistema à medida que afetam diretamente a capacidade de reprodução e de regeneração das árvores (Githiru et al. 2002), sendo que, em florestas tropicais, a grande maioria das plantas depende diretamente da efetiva dispersão por vertebrados frugívoros (Tabarelli \& Peres 2002).

As areias quartzosas do litoral oferecem um substrato desfavorável ao desenvolvimento de espécies vegetais (Rambo 1956), condicionando aspectos comuns à fisionomia arbórea, tais como baixo porte, com altura variando entre 6 e $12 \mathrm{~m}$, presença de muitos epífitos, variada diversidade de mirtáceas e outras espécies características, como Ficus organensis (Waechter 1985). A vegetação de Restinga exerce um papel fundamental na estabilização do substrato (Lamego 1946; Costa et al. 1984; Assumpção \& Nascimento 2000), bem como na preservação da fauna residente e migratória (Falkenberg 1999).

Assim, este estudo teve como foco o mutualismo existente entre as espécies arbóreas e as aves em uma mata de Restinga. Objetivou-se identificar alguns padrões de interações entre as comunidades de frugívoros e espécies vegetais, verificando quais espécies interagem e quais são mais importantes dentro desse sistema mutualístico, bem como verificar o comportamento sazonal das interações.

\section{Material e métodos}

Área de estudo - Este estudo se desenvolveu em uma pequena Mata de Restinga, denominada como Mato da Coruja $\left(30^{\circ} 22^{\prime} 51,1^{\prime}\right.$ 'S - 51 ${ }^{\circ} 00^{\prime} 29,0$ ' W). Com tamanho de 0,29 ha, a mata está localizada às margens da Lagoa Negra, na Praia de Fora, maior praia do Parque Estadual de Itapuã, município de Viamão, Rio Grande do Sul (Fig. 1).

$\mathrm{Na}$ área de estudo, o solo é arenoso, caracterizado por ser ácido e pela reduzida fertilidade (Scherer et al. 2005), composto por depósitos de areias quartzosas, de granulação fina e média, bem drenados, formando um complexo de Restinga com extensos campos de dunas, baixadas alagadas e capões (Rio Grande do Sul 1997). Segundo o sistema de Köppen, o clima da região é classificado como Cfa, subtropical úmido com verões quentes (Mota 1951).

Nesta praia, até o início da década de 1990, houve uma intensa ocupação humana, com casas de veranistas, que foram retiradas em 1991. Até janeiro/2006, o acesso a essa praia ficou restrito somente a pesquisadores e guardas-parque. Desde fevereiro/2006 a Praia de Fora foi reaberta para visitação pública, sendo que o entorno da Lagoa Negra, onde se insere a área de estudo, ainda tem acesso restrito.

Procedimento amostral - A composição florística do estrato arbóreo foi obtida do inventário realizado por Scherer et al. 2005 em toda a área do Mato da Coruja. A mata apresenta uma riqueza de 17 espécies arbóreas e um contexto de baixa diversidade específica (1,96 nats ind $\left.{ }^{-1}\right)$, tendo Myrcia palustris DC., Myrsine guianensis (Aubl.) Kuntze, Vitex megapotamica

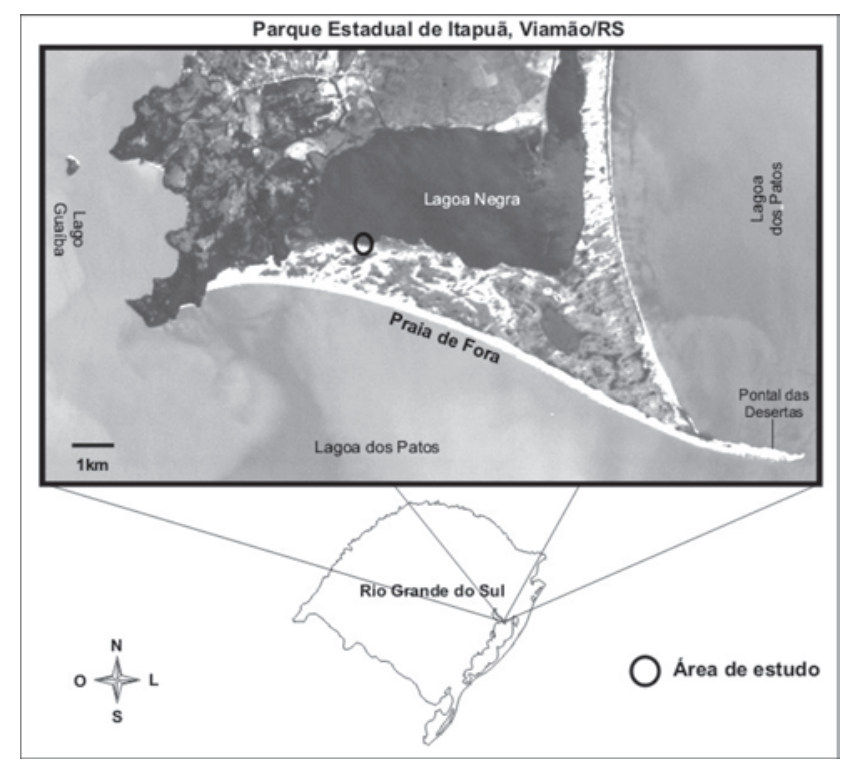

Figura 1. Localização da área de Restinga (30²2'51,1'’S $51^{\circ} 00^{\prime} 29,0$ ' $\mathrm{W}$ ) onde foram estudadas as interações entre a avifauna frugívora e o componente arbóreo no Parque Estadual de Itapuã, Viamão, RS. (Fonte da imagem de satélite Landsat: CEPSRM 2001). 
(Spreng.) Moldenke e Ficus organensis Miq. entre as espécies mais importantes na estrutura fitossociológica.

O estudo da avifauna teve amostragens semanais, com início em setembro/2003 e até agosto/2004, utilizando-se os métodos de observação visual e captura com redes de neblina ornitológicas (mist-nets). Cada rede de neblina permaneceu aberta por um período de $4 \mathrm{~h}$ e $30 \mathrm{~min}$ em cada amostragem semanal. Esse período foi multiplicado por um total de cinco redes utilizadas, obtendo-se $22 \mathrm{~h}$ e $30 \mathrm{~min}$ por saída de campo. Ao final do ano de estudo, o esforço amostral de capturas totalizou $1.106 \mathrm{~h}$.

O método de observação visual foi empregado para verificar a interação das aves com as plantas, através de transecções feitas no interior e nas bordas da Mata de Restinga, perfazendo aproximadamente seis horas contínuas de observação a partir do amanhecer em cada amostragem semanal. Em alguns dias as observações também foram feitas ao entardecer. Ao final do ano de estudo, o esforço amostral das observações visuais totalizou 357 h e 10 min. Durante as transecções, foram localizadas as espécies arbóreas que estavam frutificando e quando uma ave era avistada consumindo os frutos, foram registrados: a espécie de ave, sua guilda trófica e a espécie arbórea.

Para complementar os registros visuais das interações, foi verificada a presença de indícios de frutos nas fezes ou no regurgito de aves capturadas, que, para tanto, foram mantidas em sacos de algodão arejado por aproximadamente $20 \mathrm{~min}$. As redes ornitológicas, com malha de $22 \mathrm{~mm}$ e $7 \mathrm{~m}$ de comprimento por $3 \mathrm{~m}$ de altura, foram montadas, numa média de 5 redes por sessão de captura, no interior e na borda da mata de Restinga, sendo revisadas a cada 40 min. Os indivíduos capturados foram identificados através de literatura especializada (Bencke 2001), anilhados com anilhas padrão do Centro Nacional de Pesquisa para Conservação das Aves Silvestres (CEMAVE), tiveram seus dados biológicos e biométricos registrados e foram soltos em seguida. A identificação de frutos e partes de frutos existentes nas fezes e nos regurgitos foi feita através de comparação com frutos in natura obtidos no Mato da Coruja ou em matas adjacentes.

Além dos registros de espécies que interagiram também foram anotadas todas as espécies que utilizavam de alguma forma a Mata de Restinga, para abrigo ou forrageio, por exemplo. Com isto, obteve-se uma listagem de aves, sendo que a classificação das famílias, subfamílias e status de ocorrência seguiu
Bencke (2001) e a das guildas alimentares foi adaptada de Willis (1979). Espécies pertencentes a outras guildas que não a de frugívoros foram incluídas em onívoros, quando observadas ingerindo frutos.

Análise de dados - Para o estudo de padrões de interação entre aves e plantas foram usados somente os dados de frugivoria de maior potencial para a dispersão de sementes, excluindo-se os eventuais registros de predação, conforme indicado por Silva et al. (2002). Foi considerada como uma "interação" o registro da alimentação de uma espécie de ave em uma espécie arbórea, sendo estes dados binários, ou seja, consome ou não os frutos. Os dados de interação formaram uma matriz de aves x plantas, empregada na estimativa da conectância (Jordano 1987) e do índice de importância das espécies (Silva et al. 2002).

A conectância (C) do mutualismo entre aves e plantas é a fração registrada de todas as interações possíveis de ocorrer na comunidade considerada, sendo calculada pela seguinte fórmula:

$\mathrm{C}(\%)=\mathrm{I} /(\mathrm{F} \times \mathrm{P})$, onde $\mathrm{I}$ : número de interações registradas, $\mathrm{F}$ : número de espécies frugívoras e $\mathrm{P}$ : número de espécies vegetais, sendo $(\mathrm{F} \times \mathrm{P})$ o número total de interações possíveis de ocorrer

O índice de importância (I), criado por Murray (2000) e descrito por Silva et al. (2002), pesa a contribuição de uma espécie de ave em relação aos demais dispersores para cada uma das plantas em que ela obtém recursos alimentares. É estimado pela fórmula:

$\mathrm{I}_{j}=\Sigma\left[\left(\mathrm{C}_{i j} / \mathrm{T}_{i}\right) / \mathrm{S}\right]$, onde: $i$ é a espécie vegetal; $j$ é a espécie de ave; $\mathrm{T}_{i}$ é o número total de espécies de aves alimentando-se da espécie vegetal $i$; $\mathrm{S}$ é o número total de espécies vegetais incluídas na amostra; $\mathrm{C}_{i j}$ é 1 se a ave $j$ consome os frutos da espécie vegetal $i$ e zero se a ave não consome.

Este mesmo índice também foi usado para verificar a importância das espécies arbóreas na dieta das aves. Para tanto, $i$ foi considerado a espécie de ave e $j$, a espécie vegetal. O índice varia de zero a um.

Além disto, também foi verificado o número de eventos de consumo, isto é, o número de registros de cada espécie frugívora na comunidade arbórea observada, assim como as espécies vegetais mais consumidas. Esses dados, agregados ao número de interações por espécie, foram usados para elaborar a rede de interação planta-frugívoros (Bascompte et al. 2003; Jordano et al. 2003). A ordenação das espécies na rede foi feita usando-se valores decrescentes do número de registros. 
Foi verificada a ocorrência de variações sazonais nos mutualismos observados, avaliando-se o número médio de eventos de frugivoria (visitas), o número médio de espécies frugívoras e de espécies arbóreas consumidas por estação do ano. Os dados foram submetidos a testes de hipótese por meio da análise da variância $(\alpha=0,05)$ via aleatorização (Manly 1991; Pillar \& Orlóci 1996), com delineamento completamente casualizado, com um fator de agrupamento (estação) para as unidades amostrais (meses). Para tanto, foi utilizado o programa Multiv versão 2.1.1 (Pillar 2001).

\section{Resultados}

Das espécies do componente arbóreo do Mato da Coruja (Scherer et al. 2005) (Tab. 1), 50\% participaram das interações observadas, somando-se Erythroxylum argentinum e Guapira opposita, que não pertenciam ao estrato arbóreo adulto da mata, mas sim como plântulas, além de Eugenia hiemalis e Opuntia monacantha que apresentam aspecto arbustivo (Fig. 2). Para o grupo das aves, foram registradas 69 espécies que utilizaram de alguma forma a mata, como abrigo e/ou forrageio (Tab. 2). Dessas, 22 são espécies de aves que apresentam potencial de dispersão de sementes, ou seja, que consumiram frutos, descartando-se aquelas que inviabilizam as sementes consumidas, tais como membros de Columbidae (Tab. 2). Apenas 18 aves foram constatadas interagindo com as espécies arbóreas (Fig. 2). Da rede de interações (Fig. 2), além dessas 18 espécies de aves, fizeram parte 11 espécies vegetais, com uma conectância de $25,3 \%$, ou seja, das 198 interações possíveis de ocorrer, foram registradas apenas 50 . As espécies de aves que apresentaram maior número de visitas nas árvores com frutos foram Turdus amaurochalinus e $T$. rufiventris, totalizando aproximadamente $45 \%$ dos registros (Fig. 2). Essas espécies também foram as que interagiram com maior número de espécies arbóreas, totalizando cada uma sete interações, como demonstrado na rede de interação planta-frugívoros (Fig. 2).

Por sua vez, as espécies vegetais registradas com maior número de eventos de consumo de frutos pelas aves foram Ocotea pulchella e Myrsine spp., perfazendo cerca de $55 \%$ dos registros. M. guianensis e M. umbellata foram agrupadas por não ser possível a separação de ambas através das sementes regurgitadas ou defecadas pelas aves. Ficus organesis com 10 interações e Myrsine spp. com nove, foram as que mais interagiram com espécies dispersoras (Fig. 2).

T. amaurochalinus e T. rufiventris foram as aves com maior índice de importância (Fig. 3), caracterizando-se como as principais dispersoras em potencial do componente arbóreo da mata analisada. Essas espécies pertencem à família Muscicapidae,

Tabela 1. Espécies arbóreas da Mata da Coruja, Restinga do Parque Estadual de Itapuã, Viamão, RS (adaptado de Scherer et al. 2005) com acréscimo das espécies observadas no presente estudo.

\begin{tabular}{|c|c|}
\hline Família/Espécie & Nome popular \\
\hline \multicolumn{2}{|l|}{ CACTACEAE } \\
\hline Cereus hildmannianus K. Schum. & tuna \\
\hline Opuntia monacantha (Willd.) Haw. ${ }^{1}$ & palmatória \\
\hline \multicolumn{2}{|l|}{ ERYTHROXYLACEAE } \\
\hline Erythroxylum argentinum O.E. Schulz ${ }^{2}$ & cocão \\
\hline \multicolumn{2}{|c|}{ EUPHORBIACEAE } \\
\hline $\begin{array}{l}\text { Sebastiania serrata (Baill. ex Müll. } \\
\text { Arg.) Müll. Arg. }\end{array}$ & branquilho \\
\hline \multicolumn{2}{|l|}{ LAMIACEAE } \\
\hline $\begin{array}{l}\text { Vitex megapotamica (Spreng.) } \\
\text { Moldenke }\end{array}$ & tarumã \\
\hline \multicolumn{2}{|l|}{ LAURACEAE } \\
\hline Ocotea pulchella Mart. & canela-lageana \\
\hline \multicolumn{2}{|l|}{ MORACEAE } \\
\hline Ficus organensis Miq. & figueira \\
\hline \multicolumn{2}{|l|}{ MYRSINACEAE } \\
\hline Myrsine guianensis (Aubl.) Kuntze & capororoca \\
\hline M. umbellata Mart. & capororoca \\
\hline \multicolumn{2}{|l|}{ MYRTACEAE } \\
\hline Eugenia hiemalis Cambess. ${ }^{1}$ & guamirim-burro \\
\hline E. myrcianthes Nied. & pessegueiro-do-mato \\
\hline E. uruguayensis Cambess. & guamirim \\
\hline Myrcia palustris DC. & guamirim \\
\hline Myrciaria cuspidata O. Berg & camboim \\
\hline \multicolumn{2}{|l|}{ NYCTAGINACEAE } \\
\hline Guapira opposita (Vell.) Reitz ${ }^{2}$ & maria-mole \\
\hline \multicolumn{2}{|l|}{ RUBIACEAE } \\
\hline Randia armata (Sw.) DC. & limoeiro-do-mato \\
\hline \multicolumn{2}{|l|}{ RUTACEAE } \\
\hline Zanthoxylum fagara (L.) Sarg. & mamica-de-cadela \\
\hline \multicolumn{2}{|l|}{ SALICACEAE } \\
\hline Casearia sylvestris Sw. & chá-de-bugre \\
\hline \multicolumn{2}{|l|}{ SAPINDACEAE } \\
\hline Dodonaea viscosa (L.) Jacq. & vassoura-vermelha \\
\hline \multicolumn{2}{|l|}{ SAPOTACEAE } \\
\hline $\begin{array}{l}\text { Chrysophyllum marginatum (Hook. } \\
\text { \& Arn.) Radlk. }\end{array}$ & aguaí \\
\hline $\begin{array}{l}\text { Sideroxylon obtusifolium (Humb. ex } \\
\text { Roem. \& Schult.) T.D. Penn. }\end{array}$ & coronilha \\
\hline \multicolumn{2}{|l|}{ SOLANACEAE } \\
\hline Solanum pseudoquina A. St.-Hil. & canema \\
\hline
\end{tabular}

Espécies inseridas na listagem de Scherer et al. (2005): 'Espécies com aspecto arbustivo; ${ }^{2}$ Espécies apenas com indivíduos no estágio de plântula. 
possuindo apenas três espécies observadas, mas quando somadas representam cerca de $47 \%$ no total do índice de importância. A família Tyrannidae apresentou o maior número de espécies (5), mas teve somente 19,4\% no índice de importância.

Para as espécies de aves, F. organensis e Myrsine spp. foram as mais importantes na dieta
(Fig. 4), visto que as espécies que as consumiram não tiveram uma dieta tão variada quando comparadas com os frugívoros que se alimentaram de $O$. pulchella, que foi a mais visitada.

Houve um aumento na quantidade de eventos de frugivoria por estação do ano, com um pico significativo na primavera (Fig. 5). Além disso, verificou-se um

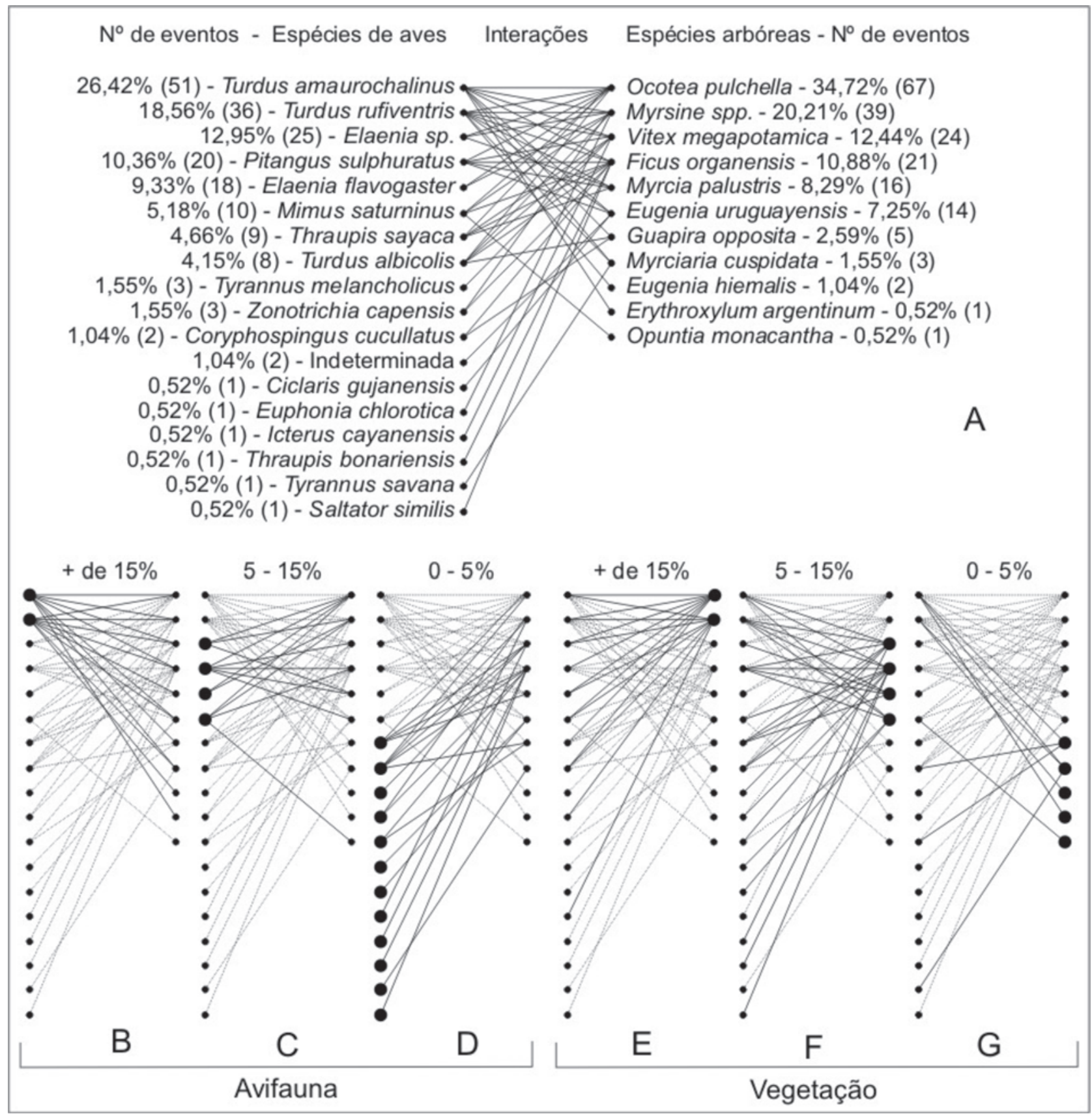

Figura 2. Rede de interações mutualísticas na comunidade de Restinga do Parque Estadual de Itapuã, Viamão, RS. Espécies ordenadas pelo número de eventos de consumo de frutos. A) Rede geral de interações e número de eventos. B, C e D) Destaque para as espécies de aves que participaram em maior e menor proporção nas interações. E, F e G) Destaque para as espécies arbóreas que participaram em maior e menor proporção nas interações. 
Tabela 2. Avifauna observada na Mata da Coruja, Restinga do Parque Estadual de Itapuã, Viamão, RS. (SO: status de ocorrência; R: residente ao longo de todo o ano; M: migratório, residente de primavera/verão, nidificando no Rio Grande do Sul; GT: guilda trófica; CD: carnívoros diurnos; GB: granívoros de borda; GCN: grandes carnívoros noturnos; GFOC: grandes frugívoros e onívoros de copa; GIS: grandes insetívoros de solo; IA: insetívoros aéreos; IB: insetívoros de borda; IN: insetívoros noturnos; INI: insetívoros de nível intermediário; ITG: insetívoros de troncos e galhos; NECR: necrófagos; NECT: nectarívoros; OB: onívoros de borda; PIC: pequenos insetívoros de copa; PIS: pequenos insetívoros de solo; PISB: pequenos insetívoros de sub-bosque; POC: pequenos onívoros de copas; POSB: pequenos onívoros de sub-bosque).

\begin{tabular}{|c|c|c|c|}
\hline Família-Subfamília/Espécie ${ }^{1}$ & Nome popular & $\mathrm{SO}^{1}$ & $\mathrm{GT}^{2}$ \\
\hline \multicolumn{4}{|l|}{ CATHARTIDAE } \\
\hline Coragyps atratus (Bechstein, 1793) & urubu-de-cabeça-preta & $\mathrm{R}$ & NECR \\
\hline \multicolumn{4}{|l|}{ ACCIPITRIDAE } \\
\hline Circus buffoni (Gmelin, 1788) & gavião-do-banhado & $\mathrm{R}$ & $\mathrm{CD}$ \\
\hline Heterospizias meridionalis (Latham, 1790) & gavião-caboclo & $\mathrm{R}$ & $\mathrm{CD}$ \\
\hline Buteo magnirostris (Gmelin, 1788) & gavião-carijó & $\mathrm{R}$ & $\mathrm{CD}$ \\
\hline Buteogallus urubitinga (Gmelin, 1788) & gavião-negro & $\mathrm{R}$ & $\mathrm{CD}$ \\
\hline \multicolumn{4}{|l|}{ FALCONIDAE } \\
\hline Caracara plancus (Miller, 1777) & caracará & $\mathrm{R}$ & $\mathrm{CD}$ \\
\hline Milvago chimachima (Vieillot, 1816) & carrapateiro & $\mathrm{R}$ & $\mathrm{CD}$ \\
\hline M. chimango (Vieillot, 1816) & chimango & $\mathrm{R}$ & $\mathrm{CD}$ \\
\hline Falco sparverius Linnaeus, 1758 & quiriquiri & $\mathrm{R}$ & $\mathrm{CD}$ \\
\hline \multicolumn{4}{|l|}{ RALLIDAE } \\
\hline Aramides saracura (Spix, 1825) & saracura-do-brejo & $\mathrm{R}$ & GIS \\
\hline \multicolumn{4}{|l|}{ COLUMBIDAE } \\
\hline Columba picazuro Temminck, 1813 & asa-branca ou pombão & $\mathrm{R}$ & GB \\
\hline Zenaida auriculata (Des Murs, 1847) & pomba-de-bando & $\mathrm{R}$ & GB \\
\hline Leptotila verreauxi (Banaparte, 1855) & juriti-pupu & $\mathrm{R}$ & GB \\
\hline L. rufaxilla (Richard \& Bernard, 1792) & juriti-gemedeira & $\mathrm{R}$ & GB \\
\hline \multicolumn{4}{|l|}{ PSITTACIDAE } \\
\hline Myiopsitta monachus (Banaparte, 1855) & caturrita & $\mathrm{R}$ & GFOC \\
\hline \multicolumn{4}{|l|}{ CUCULIDAE } \\
\hline Coccyzus melacoryphus Vieillot, 1817 & papa-lagarta-verdadeiro & M & INI \\
\hline Guira guira (Gmelin, 1788) & anu-branco & $\mathrm{R}$ & INI \\
\hline \multicolumn{4}{|l|}{ STRIGIDAE } \\
\hline Bubo virginianus (Gmelin, 1788) & jacurutu & $\mathrm{R}$ & $\mathrm{GCN}$ \\
\hline \multicolumn{4}{|l|}{ CAPRIMULGIDAE } \\
\hline Hydropsalis torquata (Gmelin, 1789) & bacurau-tesoura & $\mathrm{R}$ & IN \\
\hline \multicolumn{4}{|l|}{ TROCHILIDAE } \\
\hline Chlorostilbon aureoventris (d'Orbigny \& Lafresnaye, 1838) & besourinho-de-bico-vermelho & $\mathrm{R}$ & NECT \\
\hline Hylocharis chrysura (Shaw, 1812) & beija-flor-dourado & $\mathrm{R}$ & NECT \\
\hline \multicolumn{4}{|l|}{ PICIDAE } \\
\hline Colaptes melanochloros (Gmelin, 1788) & pica-pau-verde-barrado & $\mathrm{R}$ & ITG \\
\hline C. campestris (Vieillot, 1818) & pica-pau-do-campo & $\mathrm{R}$ & ITG \\
\hline \multicolumn{4}{|l|}{ FURNARIIDAE } \\
\hline Furnarius rufus (Gmelin, 1788) & joão-de-barro & $\mathrm{R}$ & IB \\
\hline Synallaxis spixi Sclater, 1856 & joão-teneném & $\mathrm{R}$ & IB \\
\hline Cranioleuca obsoleta (Reichenbach, 1853) & arredio-oliváceo & $\mathrm{R}$ & INI \\
\hline \multicolumn{4}{|l|}{ CONOPOPHAGIDAE } \\
\hline Conopophaga lineata (Wied-Neuwied, 1831) & chupa-dente & $\mathrm{R}$ & PIS \\
\hline \multicolumn{4}{|l|}{ TYRANNIDAE } \\
\hline Camptostoma obsoletum (Temminck, 1824) & risadinha & $\mathrm{R}$ & POC \\
\hline Elaenia $\mathrm{sp.}$ & & $\mathrm{R}$ & OB \\
\hline Elaenia flavogaster (Thunberg, 1822) & guaracava-de-barriga-amarela & $\mathrm{R}$ & OB \\
\hline Serpophaga subcristata (Vieillot, 1817) & alegrinho & $\mathrm{R}$ & IB \\
\hline Phylloscartes ventralis (Temminck, 1824) & borboletinha-do-mato & $\mathrm{R}$ & IB \\
\hline Todirostrum plumbeiceps Lafresnaye, 1846 & tororó & $\mathrm{R}$ & IB \\
\hline Lathrotriccus euleri (Cabanis, 1868) & enferrujado & M & PISB \\
\hline Xolmis irupero (Vieillot, 1823) & noivinha & $\mathrm{R}$ & IA \\
\hline Satrapa icterophrys (Vieillot, 1818) & suirirí-pequeno & $\mathrm{R}$ & IB \\
\hline Myiarchus sp. & & $\mathrm{R}$ & $\begin{array}{c}\text { PIC } \\
\text { continua }\end{array}$ \\
\hline
\end{tabular}


Tabela 2 (continuação)

\begin{tabular}{|c|c|c|c|}
\hline Família-Subfamília/Espécie ${ }^{1}$ & Nome popular & $\mathrm{SO}^{1}$ & $\mathrm{GT}^{2}$ \\
\hline Pitangus sulphuratus (Linnaeus, 1766) & bem-te-vi & $\mathrm{R}$ & OB \\
\hline Tyrannus melancholicus Vieillot, 1819 & suirirí & M & POC \\
\hline T. savana Vieillot, 1808 & tesourinha & M & POC \\
\hline \multicolumn{4}{|l|}{ HIRUDINIDAE } \\
\hline Tachycineta leucorrhoa (Vieillot, 1817) & andorinha-de-testa-branca & $\mathrm{R}$ & IA \\
\hline Progne tapera (Linnaeus, 1766) & andorinha-do-campo & M & IA \\
\hline P. chalybea (Gmelin, 1789) & andorinha-doméstica-grande & M & IA \\
\hline Notiochelidon cyanoleuca (Vieillot, 1817) & andorinha-pequena-de-casa & $\mathrm{R}$ & IA \\
\hline \multicolumn{4}{|l|}{ TROGLODYTIDAE } \\
\hline Troglodytes musculus Naumann, 1823 & corruíra & $\mathrm{R}$ & IB \\
\hline \multicolumn{4}{|l|}{ MIMIDAE } \\
\hline Mimus saturninus (Lichtenstein, 1823) & sabiá-do-campo & $\mathrm{R}$ & OB \\
\hline \multicolumn{4}{|l|}{ MUSCICAPIDAE } \\
\hline \multicolumn{4}{|l|}{ TURDINAE } \\
\hline Turdus rufiventris Vieillot, 1818 & sabiá-laranjeira & $\mathrm{R}$ & OB \\
\hline T. amaurochalinus Cabanis, 1850 & sabiá-poca & $\mathrm{R}$ & OB \\
\hline T. albicollis Vieillot, 1818 & sabiá-coleira & $\mathrm{R}$ & POSB \\
\hline \multicolumn{4}{|l|}{ EMBERIZIDAE } \\
\hline \multicolumn{4}{|l|}{ EMBERIZINAE } \\
\hline Zonotrichia capensis (Müller, 1776) & tico-tico & $\mathrm{R}$ & $\mathrm{OB}$ \\
\hline Poospiza lateralis (Nordmann, 1835) & quete & $\mathrm{R}$ & GB \\
\hline Sicalis flaveola (Linnaeus, 1766) & canário-da-terra-verdadeiro & $\mathrm{R}$ & GB \\
\hline Coryphospingus cucullatus (Müller, 1776) & tico-tico-rei & $\mathrm{R}$ & GB \\
\hline \multicolumn{4}{|l|}{ CARDINALINAE } \\
\hline Saltator similis d'Orbigny \& Lafresnaye, 1837 & trinca-ferro-verdadeiro & $\mathrm{R}$ & $\mathrm{OB}$ \\
\hline \multicolumn{4}{|l|}{ THRAUPINAE } \\
\hline Thraupis sayaca (Linnaeus, 1766) & sanhaçu-cinzento & $\mathrm{R}$ & POC \\
\hline T. bonariensis (Gmelin, 1789) & sanhaçu-papa-laranja & $\mathrm{R}$ & POC \\
\hline Stephanophorus diadematus (Temminck, 1823) & sanhaçu-frade & $\mathrm{R}$ & POC \\
\hline Pipraeidea melanonota (Vieillot, 1819) & saíra-viúva & $\mathrm{R}$ & POC \\
\hline Euphonia chlorotica (Linnaeus, 1766) & fim-fim & $\mathrm{R}$ & POC \\
\hline E. pectoralis (Latham, 1802) & gaturamo-serrador & $\mathrm{R}$ & POC \\
\hline \multicolumn{4}{|l|}{ TERSININAE } \\
\hline Tersina viridis (Illiger, 1811) & saí-andorinha & M & POC \\
\hline \multicolumn{4}{|l|}{ PARULIDAE } \\
\hline Parula pitiayumi (Vieillot, 1817) & mariquita & $\mathrm{R}$ & PIC \\
\hline Geothlypis aequinoctialis (Gmelin, 1789) & pia-cobra & $\mathrm{R}$ & PIC \\
\hline Basileuterus cullcivorus (Deppe, 1830) & pula-pula & $\mathrm{R}$ & PISB \\
\hline B. leucoblepharus (Vieillot, 1817) & pula-pula-assobiador & $\mathrm{R}$ & PISB \\
\hline \multicolumn{4}{|l|}{ VIREONIDAE } \\
\hline Cyclarhis gujanensis (Gmelin, 1789) & gente-de-fora-vem & $\mathrm{R}$ & POC \\
\hline \multicolumn{4}{|l|}{ ICTERIDAE } \\
\hline lcterus cayanensis (Linnaeus, 1766) & encontro & $\mathrm{R}$ & OB \\
\hline Oreopsar badius (Vieillot, 1819) & vira-bosta & $\mathrm{R}$ & PIC \\
\hline Molothrus bonariensis (Gmelin, 1789) & asa-de-telha & $\mathrm{R}$ & PIC \\
\hline
\end{tabular}

${ }^{1}$ Segundo Bencke (2001). ${ }^{2}$ Adaptado de Willis (1979); espécies pertencentes a outras guildas que não a de frugívoros foram incluídas em onívoros quando observadas ingerindo frutos.

aumento nas espécies frugívoras na primavera e no verão, em relação às demais estações. O número de espécies arbóreas visitadas também apresentou um incremento ao longo do ano, verificado a partir dos meses de inverno, atingindo maiores valores na primavera.

\section{Discussão}

O número reduzido de espécies interagindo reflete a baixa diversidade encontrada em matas de Restinga. Segundo Olesen \& Jordano (2002), o nível local da riqueza de espécies afeta cada espécie e suas 
interações bióticas. Em interações mutualísticas entre plantas e animais, as espécies constituem o nicho uma das outras e, se mais espécies vegetais acumulam em uma dada área, o espectro de recursos para os animais frugívoros também aumenta.

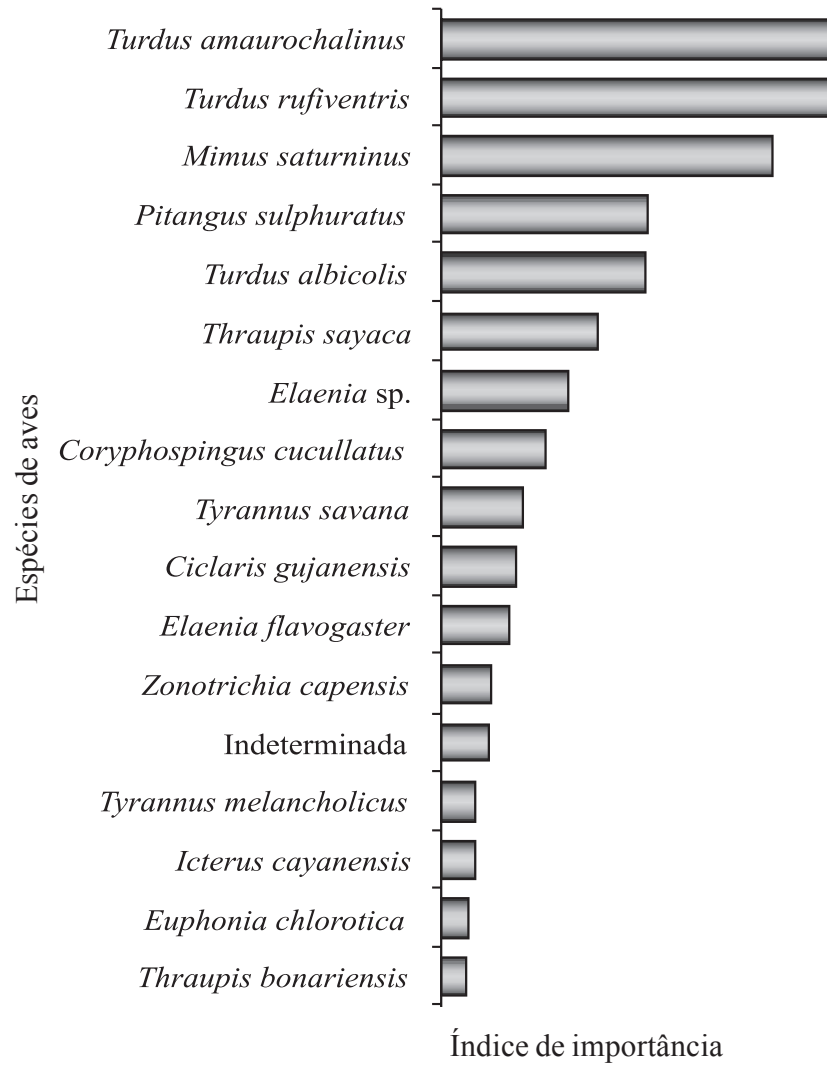

Figura 3. Importância das aves como potenciais dispersores de sementes do componente arbóreo numa Floresta de Restinga no Parque Estadual de Itapuã, Viamão, RS.

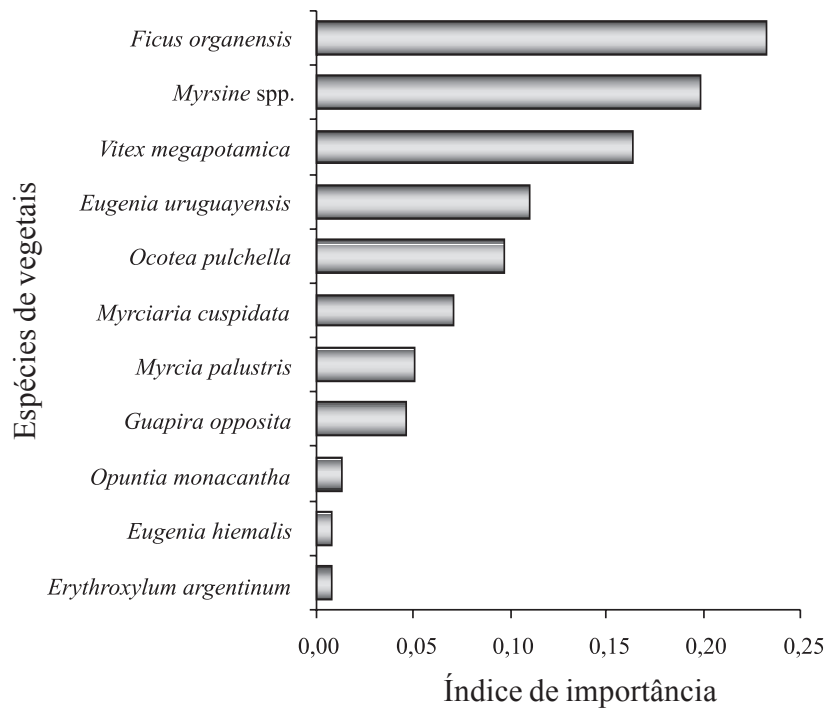

Figura 4. Importância das espécies arbóreas na dieta das aves frugívoras numa Floresta de Restinga no Parque Estadual de Itapuã, Viamão, RS.
As espécies registradas interagindo enquadramse num sistema de dispersão generalista, no qual as aves se alimentam com frutos de várias espécies e complementam sua dieta com alimentos de origem animal, além de apresentarem um tamanho corporal geralmente pequeno. As espécies vegetais desse sistema, segundo Howe (1993), apresentam como características pequenas sementes, alta fecundidade anual, taxa de remoção dos frutos reduzida e variável e alta exposição dos frutos.

O padrão mutualístico evidenciado pela rede de interações no Mato da Coruja mostra um modelo que não varia, onde poucas espécies apresentam muitas ligações e vice-versa, reforçando dados já descritos para outras comunidades vegetais neotropicais, como Restinga (M. Argel-de-Oliveira, dados não publicados), Florestas Estacionais (Montaldo 2000), Cerrado (Francisco \& Galetti 2002) e Floresta Atlântica (Silva et al. 2002). Esse modelo de interações facultativas generalistas é uma constante, com relações normalmente fracas, considerando os efeitos mútuos entre animais e plantas (Jordano 1987).

A baixa conectância encontrada reforça a ausência de interação entre determinadas espécies, o que, de acordo com Jordano (1987), pode ser devido à falta de pareamento da frutificação com a presença das espécies frugívoras, limitação por tamanho, limitações estruturais das plantas e causas não evidentes. O tamanho do dispersor, a fisiologia digestiva, a apresentação do fruto, a concentração de nutrientes e atrativos químicos e visuais afetam a escolha dos frutos, determinando as visitas (Schupp 1993).

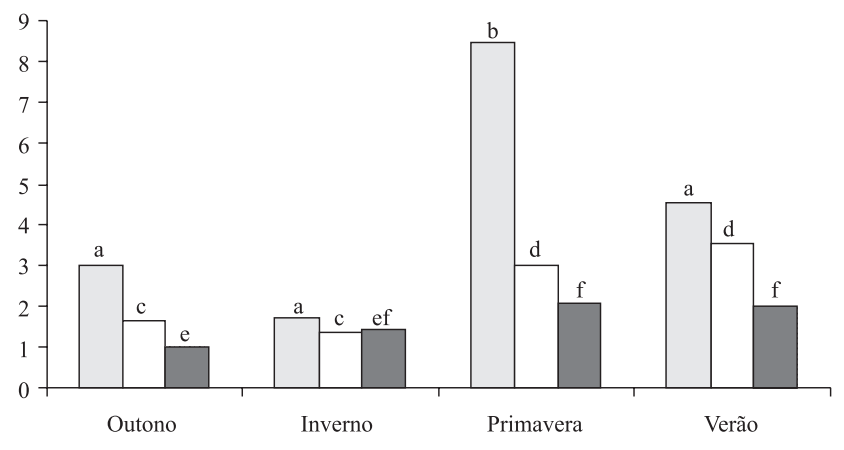

Figura 5. Variações sazonais nas interações mutualísticas entre aves frugívoras e o componente arbóreo numa Floresta de Restinga no Parque Estadual de Itapuã, RS. Barras seguidas de letras iguais, dentro de cada característica analisada, não diferem significativamente $(\mathrm{p} \leq 0,05)$. Características: $\square=$ Eventos de frugivoria; $\square=$ Espécies de aves que interagiram; $\square=$ Espécies de vegetais visitadas. 
Francisco \& Galetti (2002) relatam que nem sempre os frugívoros especialistas garantem uma dispersão mais eficiente em relação aos generalistas, sendo que muitas vezes espécies oportunistas garantem altas freqüências de visitas e taxas de consumo, além de permanecerem por curtos períodos sobre as plantas. Nesse sentido, o padrão observado no Mato da Coruja, em que a grande maioria das espécies de aves ou plantas participa de poucas interações, são as aves frugívoras pertencentes às famílias Tyranidae, Muscicapidae e Emberezidae que frequentemente contribuem com a maior proporção de interações, como também constatado em outros estudos (Argelde-Oliveira, não publicado; Montaldo 2000; Silva et al. 2002; Francisco \& Galetti 2002).

O maior trânsito pelo Mato da Coruja e o consumo de uma maior variedade de frutos condicionam Turdus amaurochalinus e T. rufiventris como elementos fundamentais na estrutura dinâmica da Mata de Restinga estudada. Estudos realizados em outros ecossistemas mostram que espécies do gênero Turdus também aparecem entre as mais importantes para a dispersão de sementes (Corlett 1998; Francisco \& Galetti 2002; Silva et al. 2002).

Em relação à importância dos frutos na dieta das aves, $F$. organensis e Myrsine spp. foram as mais relevantes, pois os frugívoros que se alimentaram dessas espécies tiveram uma dieta menos variada em relação àqueles que consumiram $O$. pulchella, que teve o maior número de eventos de consumo. Os frutos de figueiras têm se mostrado relevantes para os frugívoros, especialmente durante as estações em que frutos de outras espécies tendem a ficar escassos (Marcondes-Machado et al. 1994; Ragusa Netto 2002), enquadrando as figueiras como espécies-chave, sendo mesmo constatado para Myrsine spp. (Pineschi 1990).

$\mathrm{O}$ aumento no número de eventos de frugivoria ao longo do ano está relacionado a um incremento na frutificação e na presença de aves migratórias, principalmente da família Tyrannidae. As comunidades de aves frugívoras podem mudar sua composição de espécies e dinâmica de acordo com variações espaciais e temporais nos componentes do hábitat (Levey 1988; Loiselle \& Blake 1991), incluindo-se as variações nos padrões fenológicos da comunidade.

Espécies que não apareceram no inventário florístico (Scherer et al. 2005), como G. opposita, ou que eram jovens demais para frutificar, como E. argentinum (A. Scherer, dados não publicados), foram registradas interagindo através das fezes de aves capturadas. Com isso verificou-se um fluxo de novas espécies para o Mato da Coruja, favorecendo um potencial aumento na quantidade de espécies arbóreas nessa comunidade de Restinga.

Com os resultados obtidos, foi possível concluir que a comunidade arbórea da mata de Restinga estudada não depende de espécies especialistas em comer frutos. Os grandes responsáveis pela manutenção da dispersão de sementes nessa comunidade são espécies generalistas, fato evidenciado pelo maior número de aves com comportamento oportunista encontradas consumindo frutos. Assim, juntamente com a baixa conectância encontrada, podese sugerir que as interações mutualísticas entre aves frugívoras e árvores na Restinga do Parque Estadual de Itapuã ainda não esteja bem constituída.

\section{Agradecimentos}

Ao Prof. Andreas Kindel, pelos comentários e sugestões para realização do presente estudo; à Administração do Parque Estadual de Itapuã por disponibilizar a infra-estrutura do parque; à CAPES, pela bolsa de mestrado concedida ao primeiro autor.

\section{Referências}

Assumpção, J. \& Nascimento, M.T. 2000. Estrutura e composição florística de quatro formações vegetais de Restinga no complexo lagunar Grussaí/Iquipari, São João da Barra, RJ, Brasil. Acta Botanica Brasilica 14(3): 301-315.

Bascompte, J.; Jordano, P.; Melián, C.J. \& Olesen, J.M. 2003. The nested assembly of plant-animal mutualistic networks. Proceedings of the National Academy of Sciences of the USA 100: 9383-9387.

Bencke, G.A. 2001. Lista de referência das aves do Rio Grande do Sul. Porto Alegre, Fundação Zoobotânica do Rio Grande do Sul.

CEPSRM (Centro Estadual de Pesquisas em Sensoriamento Remoto e Meteorologia). 2001. Projeto Mosaico de Imagem do Rio Grande do Sul. Porto Alegre: UFRGS. Disponível em: <http://www.sct.rs.gov.br/programas/ mosaico/index.htm>. (Acesso em: 15/06/2004).

Corlett, R.T. 1998. Frugivory and seed dispersal by vertebrates in the Oriental (Indomalayan) Region. Biological Reviews 73: 413-448.

Corlett, R.T. \& Lucas, P.W. 1990. Alternative seed-handling strategies in primates: seed-spitting by long-tailed macaques (Macaca fascicularis). Oecologia 82: 166-171.

Costa, C.S.B.; Seeliger, U. \& Cordazzo, C. V. 1984. Aspectos da ecologia populacional do Panicum racemosum (Spreng) nas dunas costeiras do Rio Grande do Sul, Brasil. Pp. 395-411. In: L.D. Lacerda; D.S.D. Araújo; R. Cerqueira \& B. Turcq (eds.). Restingas: Origem, Estrutura e Processos. Niterói, CEUFF. 
Falkenberg, D.B. 1999. Aspectos da flora e da vegetação secundária da Restinga de Santa Catarina, Sul do Brasil. Insula 28: 1-30.

Francisco, M.R. \& Galetti, M. 2002. Aves como potenciais dispersoras de sementes de Ocotea pulchella (Lauraceae) numa área de vegetação de cerrado do sudeste brasileiro. Revista Brasileira de Botânica 25(1): 11-17.

Githiru, M.; Lens, L.; Bennur, L.A. \& Ogol, C.P.K.O. 2002. Effects of site and fruit size on the composition of avian frugivore assemblages in a fragmented Afrotropical forest. Oikos 96(2): 320-330.

Howe, H.F. 1977. Bird activity and seed dispersal of a tropical wet forest tree. Ecology 58: 539-550.

Howe, H.F. 1981. Dispersal of a neotropical nutmeg (Virola sebifera) by birds. Auk 98(1): 88-98.

Howe, H.F. 1993. Specialized and generalized dispersal systems: where does 'the paradigm' stand? Vegetatio 107/108: 3-13.

Howe, H.F. \& Smallwood, J. 1982. Ecology of seed dispersal. Annual Review of Ecology and Systematics 13: 201-228.

Howe, H.F. \& Vande Kerckhove, G.A. 1981. Removal of wild nutmeg (Virola surinamensis) crops by birds. Ecology 62: 1093-1106.

Jordano, P. 1987. Patterns of mutualistic interactions in pollination and seed dispersal: connectance, dependence asymetries, and coevolution. American Naturalist 129(5): 657-677.

Jordano, P.; Bascompte, J. \& Olesen, J.M. 2003. Invariant properties in coevolutionary networks of plant-animal interactions. Ecology Letters 6: 69-81.

Jordano, P. \& Schupp, E.W. 2000. Determinants of seed disperser effectiveness: the quantity component and patterns of seed rain for Prunus mahaleb. Ecological Monographs 70: 591-615.

Lamego, A.R. 1946. O homem e a Restinga. Rio de Janeiro, IBGE.

Levey, D.J. 1987. Seed size and fruit-handling techniques of avian frugivores. American Naturalist 129: 471-485.

Levey, D.J. 1988. Spatial and temporal variation in Costa Rican fruit and fruit-eating bird abundance. Ecological Monographs 58: 251-269.

Loiselle, B.A. \& Blake, J.G. 1991. Temporal variation in birds and fruits along an elevation gradient in Costa Rica. Ecology 72: 180-193.

Manly, B.F.J. 1991. Randomization and Monte Carlo methods in biology. London, Chapman \& Hall.

Marcondes-Machado, L.O.; Paranhos, S.J. \& Barros, Y.M. 1994. Estratégias alimentares de aves na utilização de frutos de Ficus microcarpa (Moraceae) em uma área antrópica. Iheringia, Série Zoologia 77: 57-62.

Montaldo, N.H. 2000. Exito reproductivo de plantas ornitócoras en um relicto de selva subtropical em Argentina. Revista Chilena de Historia Natural 73(3): 511-524.

Mota, F.S. 1951. Estudos do clima do Estado do Rio Grande do Sul, segundo o sistema de W. Köppen. Revista Brasileira de Geografia 13(2): 275-284.
Murray, K.G. 2000. The importance of different bird species as seed dispersers. Pp. 294-295. In: N.M. Nadkarni \& N.T. Wheelwright (eds.). Monteverde: Ecology and Conservation of a Tropical Cloud Forest. New York, Oxford University Press.

Pillar, V.D. 2001. MULTIV, Software for Multivariate Exploratory Analysis and Randomization Testing. Porto Alegre, Departamento de Ecologia, Universidade Federal do Rio Grande do Sul.

Pillar, V.D. \& Orlóci, L. 1996. On randomization testing in vegetation science: multifactor comparisons of relevé groups. Journal of Vegetation Science 7: 585-592.

Pineschi, R.B. 1990. Aves como dispersores de sete espécies de Rapanea (Myrsinaceae) no maciço do Itatiaia, estados do Rio de Janeiro e Minas Gerais. Ararajuba 1: 73-78.

Ragusa Netto, J. 2002. Fruiting phenology and consumption by birds in Ficus calyptroceras (Miq.) Miq. (Moraceae). Brazilian Journal of Biology 62(2): 339-346.

Rambo, B. 1956. A Fisionomia do Rio Grande do Sul. 2 ed. Porto Alegre, Livraria Selbach.

Rio Grande do Sul. 1997. Plano de manejo Parque Estadual de Itapuã/RS. Porto Alegre, Secretaria da Agricultura e Abastecimento, Departamento de Recursos Naturais Renováveis.

Scherer, A.; Maraschin-Silva, F. \& Baptista, L.R.M. 2005. Florística e estrutura do componente arbóreo de matas de Restinga arenosa no Parque Estadual de Itapuã, RS, Brasil. Acta Botanica Brasilica 19(4): 717-727.

Schupp, E.W. 1993. Quantity, quality and the effectiveness of seed dispersal by animals. Vegetatio 107/108: 15-29.

Silva, W.R.; De Marco-Jr, P.; Hasui, E. \& Gomes, V.S.M. 2002. Patterns of fruit-frugivore interactions in two Atlantic Forest bird communities of south-eastern Brazil: implications for conservation. Pp. 423-435. In: D.J. Levey; W.R. Silva \& M. Galetti (eds.). Seed dispersal and frugivory: ecology, evolution and conservation. New York, CAB International.

Tabarelli, M. \& Peres, C.A. 2002. Abiotic and vertebrate seed dispersal in the Brazilian Atlantic Forest: implications for forest regeneration. Biological Conservation 106: 165-176.

Terborgh, J. 1986. Community aspects of frugivory in tropical forests. Pp. 371-384. In: A. Estrada \& T.H. Fleming (eds.). Frugivores and seed dispersal. Dordrecht, W. Junk Publishers.

Waechter, J.L. 1985. Aspectos ecológicos da vegetação de Restinga no Rio Grande do Sul, Brasil. Comunicações do Museu de Ciências da PUCRS, série Botânica 33: 49-68.

Waechter, J.L. 1990. Comunidades vegetacionais das Restingas do Rio Grande do Sul. Pp. 228-248. In: Anais do Simpósio de Ecossistemas da Costa Sul e Sudeste Brasileira. Águas de Lindóia 1990. São Paulo, ACIESP. v. 3, n. 71-3.

Wheelwright, N.T. 1991. How long do fruit-eating birds stay in the plants where they feed? Biotropica 23: 29-40.

Willis, E.O. 1979. The composition of avian communities in remanescent woodlots in southern Brazil. Papéis Avulsos de Zoologia 33(1): 1-25. 